\title{
Coherent Two-Dimensional Multiphoton Photoelectron Spectroscopy of Metal Surfaces
}

\author{
Marcel Reutzel, ${ }^{*}$ Andi Li, and Hrvoje Petek ${ }^{\dagger}$ \\ Department of Physics and Astronomy and Pittsburgh Quantum Institute, \\ University of Pittsburgh, Pittsburgh, Pennsylvania 15260, USA
}

(Received 18 July 2018; revised manuscript received 11 January 2019; published 8 March 2019)

\begin{abstract}
Light interacting with high-electron-density materials elicits an ultrafast coherent many-body screening response on sub- to few-femtosecond timescales, which makes its experimental observation challenging. Here, we describe the coherent two-dimensional (2D) multiphoton photoemission (mPP; $m=2-5$ ) study of the Shockley surface (SS) state of $\operatorname{Ag}(111)$ as a benchmark for spectroscopy of the coherent nonlinear responses of metals to intense optical fields in the perturbative regime; similar 2D signatures can be expected for coherent responses in other materials like low-dimensional semiconductors and strongly correlated materials. Employing interferometric time-resolved multiphoton photoemission spectroscopy (ITR-mPP), we correlate the coherent polarizations excited in the sample with photoelectron energy distributions where the interaction terminates and photoelectrons carry away the information on their excitation. By measuring the nonresonant three- and four-photon photoemission of the SS state, as well as its replicas in above-threshold photoemission (ATP), we record the coherent response of the $\operatorname{Ag}(111)$ surface by $2 \mathrm{D}$ photoemission spectroscopy and relate it to its band structure. A 2D analysis of the SS state and its ATP replicas shows similar behavior, indicating that they are $m$ th- and $m$ th +1 -order coherent processes in a contradiction of the common attribution of ATP as a sequential process where a photoelectron excited above the vacuum level absorbs one or more additional photons. We interpret the mPP process by an optical Bloch equation model, which reproduces the main features of the surface state coherent polarization dynamics in ITR-mPP experiments: The distributions of spectroscopic components in 2D photoelectron spectra of coherent mPP are shown to follow systematically the $n / m$ ratio, where $n$ and $m$ are orders of the induced coherence and the photoemission process contributing to the signal.
\end{abstract}

DOI: 10.1103/PhysRevX.9.011044

Subject Areas: Condensed Matter Physics

\section{INTRODUCTION}

The quantum optoelectronic response of metals is dominantly coherent, as manifested by specular reflection of light, yet large bandwidths and ultrafast phase relaxation of the many-body electronic system impede its time-resolved spectroscopic investigations [1-5]. The coherent responses of metals are evident in highly nonlinear optical interactions like above-threshold multiphoton photoemission (ATP) [6-11] and high-harmonic generation (HHG) [12,13]. Highorder coherent processes epitomize the strong-field, attosecond physics of atomic or molecular gases, as well as electron emission from nanostructured metal tips [14-18]. In lightwave electronics [19,20], exemplified by HHG in solids, optical driving of petahertz currents, and imaging of singlemolecule motion by terahertz fields [21], ultrafast coherent

\footnotetext{
*mar331@pitt.edu

petek@pitt.edu
}

Published by the American Physical Society under the terms of the Creative Commons Attribution 4.0 International license. Further distribution of this work must maintain attribution to the author(s) and the published article's title, journal citation, and DOI. interactions are opening promising subjects of basic and applied research on optical field-driven manipulation of solid-state materials [22]. Atomic gas phase models for HHG [20,23] have been applied to ultrafast high-field optical responses of solids, such as photon dressing of FloquetBloch states in topological insulators [24], laser-assisted photoemission from metal surfaces [25] by attosecond streaking [26-29], high-order multiphoton photoemission (mPP) from sharp metal tips [15,18,30-32], and coherent microscopy of plasmonic fields [33-38]. Yet many coherent processes, such as the ultrafast screening of optical fields in metals [39], are unique to the solid state. Thus, the ability to probe and characterize how fields drive coherent nonlinear phenomena in the solid state $[40,41]$ enables fundamental studies of coherent control [2,42], electronic processes and interactions $[2,4]$, and quantum information processing, as well as applications such as the generation of ultrashort electron pulses [30,43,44] for time-resolved diffraction [45] and microscopy $[46,47]$ with potentially attosecond time resolution $[40,41]$.

While coherence is essential for probing and harnessing of attosecond timescale high-field quantum phenomena, its effects are more easily examined at lower fields by perturbative, nonlinear mPP spectroscopy of the surface 
and bulk electronic bands of well-ordered metals [1-4]. Detailed information on the electronic structures, as well as phase and energy relaxation dynamics at surfaces, interfaces, and complex adsorbate structures, has been obtained by time-resolved multiphoton photoemission (TR-mPP) spectroscopy [48-55]. In mPP, the combined energy of two or several photons excites electrons from the occupied bands below the Fermi level $\left(E_{F}\right)$ to the photoemission continuum above the vacuum level $\left(E_{\mathrm{vac}}\right)$, via real or virtual unoccupied intermediate states. For mPP, with $m \geq 3$, high optical field strengths are necessary to detect sufficient photoelectron yields while taking care that sample damage or space charge do not compromise the spectroscopic measurement. In addition to the lowest-order mPP signals excited with ultrashort laser pulses having perturbative optical field strengths, higher-order nonlinear effects, like ATP [6-11], can also be detected. ATP in the perturbative regime can be a sequential or a coherent process. In previous mPP measurements on atoms, molecules, and solids, it has been described, with scant evidence [6-11,56,57], as a two-step process where a photoelectron is excited and, before it escapes into the vacuum, it absorbs one or more photons to gain additional kinetic energy. It can also occur by the rectification of nonlinear polarization directly populating photoemission continuum states above the threshold for photoemission. In either case, ATP in the nonperturbative regime is different from the perturbative regime, where strong field acceleration of electrons enables them to gain ponderomotive energy $[14,58]$.

Interferometric time-resolved multiphoton photoemission (ITR-mPP) is an effective multidimensional spectroscopy for investigating quantum coherence, which has been developed and applied to study dephasing of surface and bulk bands in metals [1,48,59-61], coherence in interfacial charge transfer processes $[62,63]$, and the formation of transient excitons at the $\operatorname{Ag}(111)$ surface [39,64-66]; moreover, related techniques have been applied to vibronic dynamics in molecules [67] and in coherent photoelectron emission microscopy [33,35,37,38]. Cui et al. [39] developed and applied the multidimensional capabilities of ITR-mPP to record movies of the coherent transformation of a transient exciton formed by resonant two-photon excitation of the first image potential (IP) state $\leftarrow$ Shockley surface (SS) state transition of Ag (111) by collecting photoelectron energy $(E)$ and parallel momentum $\left(k_{\|}\right)$distributions with an imaging electron spectrometer while scanning the optical delay time $(t)$ between identical pump and probe pulses with approximately 50 as (suboptical cycle) resolution.

Optical phase-resolved $E\left(k_{\|}, t\right)$ movies recorded by ITR$\mathrm{mPP}$ enable the acquisition and analysis of the coherent electron dynamics in the time, frequency, energy, and momentum dimensions. Fourier transforms (FTs) of the optical-field-dependent photoelectron energy distributions with respect to the time delay for a given $k_{\|}$give two-dimensional (2D-FT) photoelectron spectra, which correlate the coherent polarization frequencies (energies) induced in the sample with the photoelectron energies that are excited. Such 2D-FT spectra record the linear and nonlinear current-current correlations and, thus, enable the analysis of coherences that produce the mPP signal [68-71]. Whereas optical spectroscopy measures energyand momentum-integrated optical transitions between states, the merit of mPP spectroscopy is that it provides the $E$ and $k_{\|}$resolved information on the photoexcitation process. Because $E$ and $k_{\|}$are conserved and resolved, the $\mathrm{mPP}$ spectra of surface bands are homogeneously broadened. Therefore, by contrast to the 2D optical spectroscopy, which educes the coherent optical response from correlations between tunable excitation and detection fields [72,73], an ITR-mPP experiment correlates the coherent polarizations and populations with the final photoelectron distribution [39]. The coherent nonlinear polarizations investigated in ITR-mPP are directly related to the HHG from metal surfaces $[70,74]$. In the perturbative regime, a field $\boldsymbol{E}$ induces a polarization $\boldsymbol{P}$ that can be described by $\boldsymbol{P}=\sum_{n=1}^{\infty} \epsilon_{0} \chi_{n} \boldsymbol{E}^{n}$, where $n$ is an integer order of the response and the susceptibility $\chi_{n}$ describes the energy, momentum, and time integrated linear and nonlinear

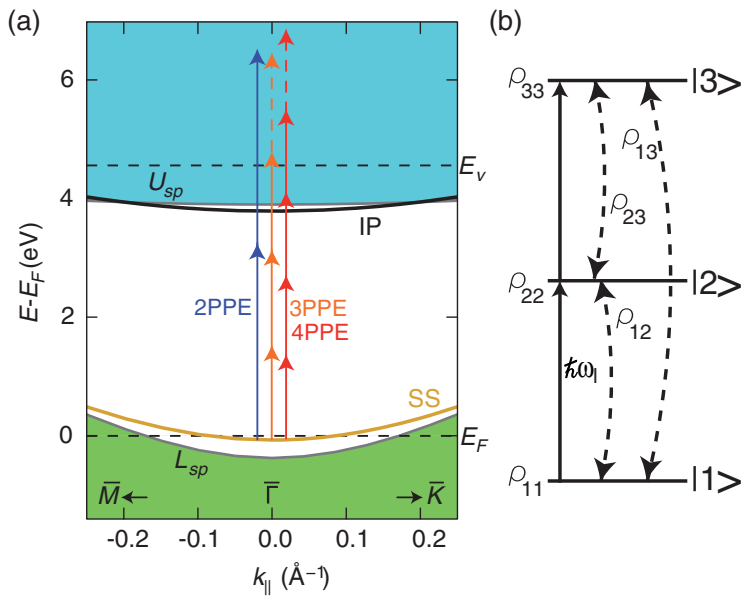

FIG. 1. (a) Surface projected band structure of $\operatorname{Ag}(111)$ after Ref. [80]. The lower $\left(L_{s p}\right)$ and the upper $\left(U_{s p}\right)$ bulk $s p$ bands are shown by green and light blue shading bracketing the projected band gap, respectively. The Shockley surface state, which is occupied for a narrow $k_{\|}$range, is plotted in ocher and the first image potential state in black. Photoemission of electrons from the SS state is induced in second-, third-, and fourth-order mPP ( $m=2,3,4)$ processes, depending on the photon energy, as indicated by the arrows. ATP in $m=(3+1)$ - and $m=(4+1)$ photon excitation is indicated by the dashed arrows. (b) Diagram of a canonical three-level system used in OBE simulations consisting of the occupied initial state $|1\rangle$, the unoccupied intermediate $|2\rangle$, and the final photoelectron state $|3\rangle$. Upward and dashed arrows indicate population transfers and coherences between the levels, respectively, which are labeled with the corresponding elements of the density matrix $\hat{\rho}$; the state energies depend on a specific electronic structure. 
system responses to the applied field [70,75]. In ITR-mPP experiments on the $\operatorname{Ag}(111)$ surface, we detect high-order energy- and momentum-resolved coherent polarizations, which drive the nonlinear response including the process of ATP.

ITR-mPP experiments have been interpreted by means of coherent 2D-FT photoelectron spectroscopy to deduce the excitation pathways that contribute to the $\mathrm{mPP}$ process $[39,63,76]$. Here, we examine the signatures of coherence in 2D-FT photoelectron spectra for nonresonant 3PP, 4PP, and 5PP, including ATP excitation, of the partially occupied SS at the pristine, noble metal $\operatorname{Ag}(111)$ surface. The wellknown surface electronic structure of $\operatorname{Ag}(111)$ [Fig. 1(a)], with a broad projected band gap at $k_{\|}=0 \AA^{-1}$, facilitates spectroscopic assignments of the nonresonant mPP and simulation of the coherent polarization dynamics by an optical Bloch equation (OBE) model [70,77-79]. The simulations reproduce the main features of the experimental 2D-FT spectra, providing insights into the coherent multiphoton excitation as well as ATP at metal surfaces, highlighting especially that ATP in the perturbative regime occurs by coherent rectification of a high-order polarization field, contradicting its common description as a two-step sequential process.

\section{METHODS}

\section{A. Experimental setup: ITR-mPP}

Interferometrically time-resolved $\mathrm{mPP}$ experiments $[39,48,61]$ are performed in an ultrahigh vacuum chamber with a base pressure of $<10^{-10}$ mbar. The $\mathrm{Ag}(111)$ surface is prepared by $\mathrm{Ar}^{+}$ion sputtering $(20 \mathrm{~min}, 1500 \mathrm{~V}, 3 \mu \mathrm{A})$ and subsequent annealing (10 $\mathrm{min}, 550 \mathrm{~K})$; its quality is judged by a sharp SS peak in the mPP spectra. The sample is cooled to approximately $90 \mathrm{~K}$ for mPP experiments to minimize dephasing by electron-phonon interaction.

mPP spectra are excited with a noncollinear optical parametric amplifier (NOPA) pumped by a Clark MXR Impulse fiber laser oscillator-amplifier system operating at a 1-MHz pulse repetition rate [81]. The fundamental and second harmonic outputs of the NOPA provide tunable ultrafast laser pulses in a 930-270 nm range with $<20$ fs pulse durations. 3PP and 4PP are excited by focusing $p$ polarized near-IR pulses at an angle of incidence of $45^{\circ}$ onto the sample with an average power of approximately $70 \mathrm{~mW}$. In this experimental geometry, $<2 \mathrm{~mW}$ is adsorbed by the sample, at a fluence of $<0.1 \mathrm{~mJ} / \mathrm{cm}^{2}$ (with an estimated beam diameter of approximately $100 \mu \mathrm{m}$ ); the time-averaged heating of the sample due to laser irradiation is $<1 K$ and can thus be neglected. In addition, we show temperature-dependent 2D-FT data in Supplemental Material (Fig. S1) [82], which shows that the temperature effect on 2D-FT spectra of the SS state component is negligible. The Keldysh parameter for the excitation, $\gamma \approx 20$, is much above $\gamma<1$, where the nonperturbative effects such as field-induced tunneling are expected to contribute [17,58]; thus, our experiments are in the perturbative regime, where the $\mathrm{mPP}$ spectra are dominantly generated by dipole coupling of the surface and bulk bands $[22,83]$. 2PP spectra with UV light are excited with an average power of approximately $5 \mathrm{~mW}$.

After compensation with negative dispersion mirrors, the NOPA pulses are characterized by recording ITR-mPP autocorrelations on the polycrystalline Ta sample holder at the location of the $\operatorname{Ag}(111)$ sample, optimizing for the maximum pulse compression (cf. Supplemental Material, Figs. S2 and S3 [82]). A further test of the dispersion compensation is provided by the SS state spectrum, whose intensity and frequency strongly depend on the laser chirp [2].

For the interferometric measurements, identical pumpprobe pulse pairs are produced with a Mach-Zehnder interferometer, and their delay is scanned by piezoelectrically varying the path length of one arm [1]. One output of the interferometer is directed onto the sample as the mPP excitation source, while the other one is transmitted through a monochromator to generate interference fringes that calibrate the scan time delay $[1,39,48]$. A hemispherical electron energy analyzer with a position sensitive electron-counting detector produces energy- and angleresolved 2D spectra. A series of spectra are taken in approximately 100 as intervals of pump-probe delay to generate $3 \mathrm{D} E\left(k_{\|}, t\right)$ movies. Multiple pump-probe scans are averaged to improve the counting statistics [39].

\section{B. OBE simulation}

We simulate the coherent ITR-mPP data with OBE simulations based on $(m+1)$-level excitation schemes; the general case for a three-level system is shown in Fig. 1(b). The OBE method describes semiclassically the optical excitation of atomic or molecular systems [77] and, thus, can include only heuristically the many-body effects associated with the quasiparticle formation and dephasing. For homogeneously broadened bands of a metal surface, the $\mathrm{mPP}$ process can be modeled by an $(m+1)$-level system, as has been verified previously $[1-3,48,78,79,84,85]$. The time evolution of the excited system is modeled by a density matrix $\hat{\rho}$, where the diagonal elements $\hat{\rho}_{i i}$ represent populations and the off-diagonal elements $\hat{\rho}_{i j}$ the coherences or polarizations generated by the optical field [cf. Fig. 1(b)]. In a nonresonant $\mathrm{mPP}$ process, the photoinduced coherences transfer populations from the initial to the final state, as described by the Liouville-von Neumann equation

$$
i \hbar \frac{\partial \hat{\rho}}{\partial t}=[\hat{H}, \hat{\rho}]+\frac{\partial \hat{\rho}_{D}}{\partial t} .
$$

$\hat{H}_{0}$ and $\hat{H}_{L M}$ in the full Hamiltonian $\hat{H}=\hat{H}_{0}+\hat{H}_{L M}$ describe the unperturbed system and semiclassically treated light-matter interaction in the dipole approximation, 
respectively. The dissipation matrix $\hat{\rho}_{D}$ phenomenologically describes the damping of the density matrix terms $[78,79]$, where the diagonal $\hat{\rho}_{D i i}$ and off-diagonal $\hat{\rho}_{D i j}$ elements represent the inelastic decay rates $1 / T_{1 i}$ of level $i$ and dephasing of the coherences $1 / T_{2 i j}^{*}$ between the levels $i$ and $j$, respectively.

In the OBE calculations, we choose the parameters of $\hat{\rho}$ and $\hat{\rho}_{D}$ as follows: The energy levels and the photon energy are defined by the experiment; the laser pulse width is set to $\tau_{l}=20 \mathrm{fs}$. The initial and the final state inelastic decay times $T_{1 i}$ are set to infinity ( $T_{1 \mathrm{SS}}=T_{1 F} \gg 1 \mathrm{fs}$ ) [79], while that for the first IP state is its measured value of $T_{1 I P}=$ $30 \mathrm{fs}$ [86]. The pure dephasing times $T_{2 i j}^{*}$ are set in a range between 15 and 1500 fs. For $m \geq 3$ (four- and five-level systems), the ladder climbing is described by virtual intermediate states (VI), for which the inelastic decay and pure dephasing times are set to $T_{1 \mathrm{VI}}=1 \mathrm{fs}$ and $T_{2 i j}^{*} \gg 1$ fs, respectively. We emphasize that the description of ITR-mPP experiments with OBE simulations neglects many-particle contributions, especially when treating higher-order $\mathrm{mPP}(m \geq 3)$, in which the IP states cannot be exclusively identified as intermediate states facilitating the $m$-photon process. The goal of the OBE simulation is not to provide a quantitative modeling of the $\mathrm{mPP}$ process but primarily to reproduce the signatures of the coherent excitation process. Hence, we do not attempt to extract quantitative information on decay and dephasing rates based on the simulation of our experiments. To model the ITR-mPP more quantitatively, collective effects, for example, screening of the optical field by the plasmonic response of the $\operatorname{Ag}(111)$ [66,70,87-89], would have to be included, which, however, cannot be accomplished by the OBE method. We note that frequency-dependent 2PP spectra of the $\operatorname{Ag}(111)$ surface indicate that the collective plasmonic response of $\mathrm{Ag}$ strongly affects the $2 \mathrm{PP}$ yields [90]; such a collective response should also affect the mPP data presented here.

\section{RESULTS AND DISCUSSION}

The occupied electronic structure of the $\operatorname{Ag}(111)$ surface is known from one-photon angle-resolved photoemission spectroscopy [91-94]. The unoccupied band structure has been studied by inverse photoemission [80] as well as 2PP and 3PP spectroscopy [86,95-102]. The primary focus of these $\mathrm{mPP}$ experiments has been the image potential state series, their excitation from the occupied SS state including the formation of an excitonic state [39], their lifetimes, and the bulk transition from the lower, $L_{s p}$, to the upper, $U_{s p}, s p$ band [39,86,95-102].

In Fig. 2, we show energy- and $k_{\|}$-resolved 2PP spectra taken by excitation with $\hbar \omega_{l}=3.32 \mathrm{eV}$ photons. 2PP spectroscopy of $\operatorname{Ag}(111)$ in this energy range is well documented in the literature; our band energies and $k_{\|}$ dispersions are in agreement with these reports $[99,100]$.

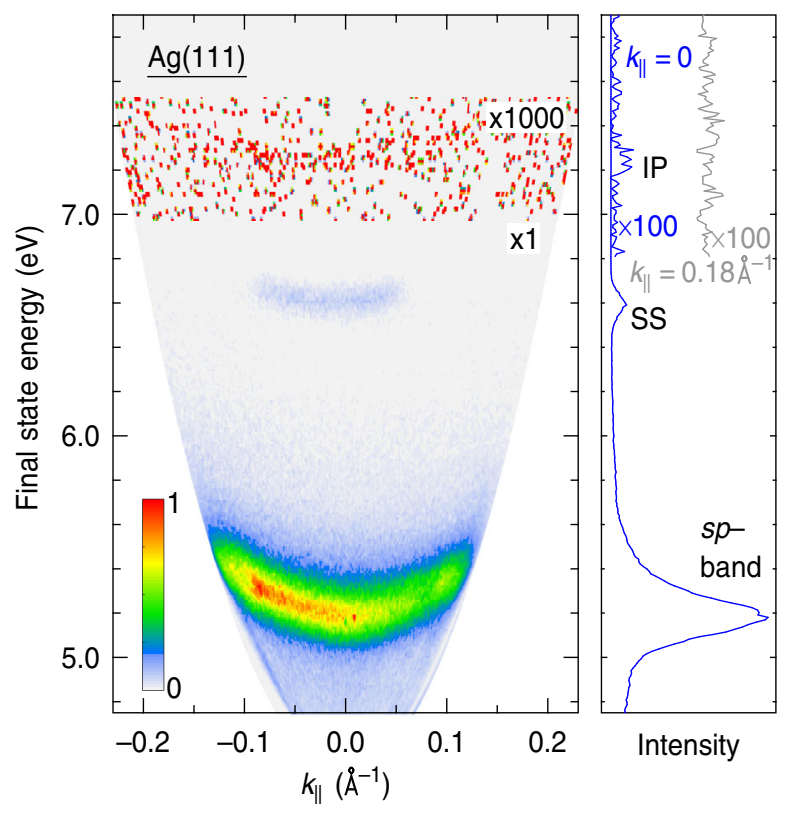

FIG. 2. Energy- and $k_{\|}$-resolved 2PP spectra of $\mathrm{Ag}(111)$ $\left[\hbar \omega_{l}=3.32 \mathrm{eV}\right.$; the corresponding excitation diagram for the SS state is given in Fig. 1(a)]; the photoemission spectral elements are labeled in the line profile (right, blue) taken at $k_{\|}=0$. The SS state and the $s p$ band are detected through coherent two-photon excitation and resonant two-photon absorption from $L_{s p}$ to $U_{s p}$, respectively. The color intensity scale represents the photoelectron counts; above $7 \mathrm{eV}$, the color scale is amplified by a factor of 1000 to show the weak contribution of the first IP state. The pixelated image results from photoelectroncounting statistics. As emphasized by the line profiles shown for $k_{\|}=0 \AA^{-1}$ (blue) and $k_{\|}=0.18 \AA^{-1}$ (gray), the IP state is detected only for the $k_{\|}$range in which the SS state is occupied, demonstrating that it is excited by a nonresonant one-photon absorption from SS.

The 2PP spectra are dominated by the SS state and the $s p$ band, which are, respectively, detected by nonresonant and resonant ( $L_{s p}$ to $U_{s p}$ ) coherent two-photon absorption; the SS state is occupied in a narrow $k_{\|}$range where it exists below $E_{F}$ [excitation diagram in Fig. 1(a)]; the $k_{\|}$asymmetry of the surface and bulk photoemission intensities are discussed in Ref. [100]. The first IP state is detuned from resonance with SS and, hence, appears with a low intensity; significantly, it appears only in the $k_{\|}$range where the SS state is occupied (compare the blue and gray line profiles in Fig. 2), which indicates that it is populated by nonresonant one-photon excitation from SS [103] rather than by resonant two-photon excitation from the bulk $L_{s p}$ band, which is possible but would not limit the $k_{\|}$range.

\section{A. OBE simulation}

Because the OBE approach has proven useful to interpret and analyze ITR-mPP data $[1,48,62,63,76,104]$, in this 

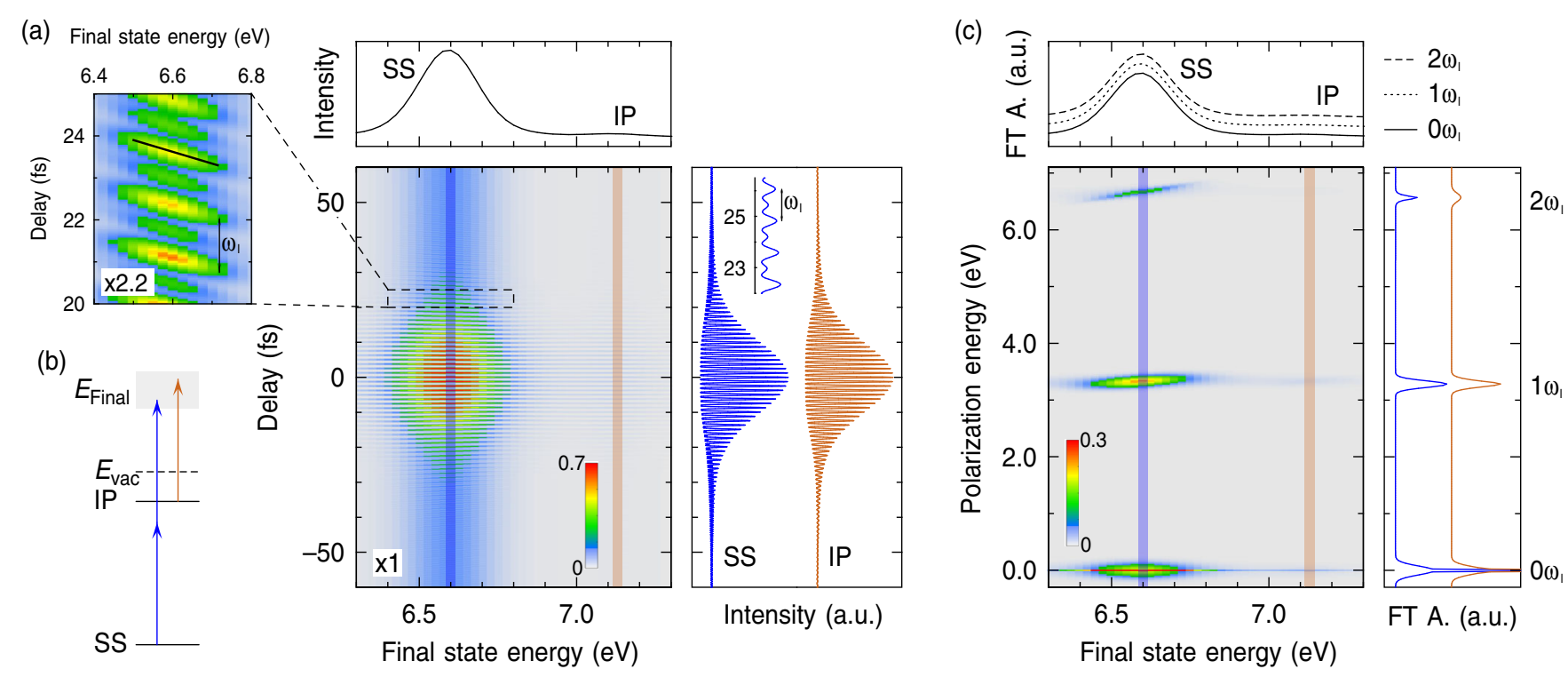

FIG. 3. (a) OBE simulation of an energy- and phase-resolved ITR-2PP measurement for the three-level system shown in (b), which consists of the occupied initial state (SS), unoccupied intermediate state (IP), and final state continuum $\left(E_{\text {final }}\right)$ above the vacuum level $E_{\text {vac }}$ (gray box). The center plot in (a) shows the calculated ITR-2PP measurement, specifically the population of the final state (color coded) as a function of the energy and pump-probe delay. Top: The calculated final state energy spectrum consisting of the SS and IP states is a profile through the ITR-2PP data at $t=0 \mathrm{fs}$. Right: Interferometric two-pulse correlation traces are profiles through ITR-2PP for the energies of SS and IP states; oscillations caused by interferences at the driving laser frequency $1 \omega_{l}$ and its second harmonic $2 \omega_{l}$ are expanded in the inset. The color-coded expanded inset of ITR-2PP shows interference fringes tilting towards $t=0 \mathrm{fs}$ with an increasing final state energy (highlighted by the black line at approximately $24 \mathrm{fs}$ ). (c) 2D-FT photoelectron spectra obtained by Fourier transformation of the time axis in (a). The polarization energies (the Fourier axis) induced in the sample at $0 \omega_{l}, 1 \omega_{l}$, and $2 \omega_{l}$ are correlated with the final state energies at which the photoemission process terminates. Top: Normalized Fourier filtered 2PP spectra at $0 \omega_{l}, 1 \omega_{l}$, and $2 \omega_{l}$ (top to bottom). Right: Line profiles taken at the SS and the IP state energies showing FT amplitudes of the $0 \omega_{l}, 1 \omega_{l}$, and $2 \omega_{l}$ oscillations.

section we present an OBE simulation of the optical coherent electronic response of the $\operatorname{Ag}(111)$ surface, by calculating the 2PP signal as the delay is varied interferometrically between identical pump and probe pulses [Fig. 3(a)]. The calculation assumes the three-level scheme in Fig. 3(b), which qualitatively models the 2PP of SS state in Fig. 2. Subsequently, we address the related coherent excitation dynamics in higher orders of multiphoton excitation by presenting the ITR-mPP $[m=3,4,5$ (ATP)] data and their OBE simulations. The surface and bulk states appear independently in the mPP spectra, and hence we do not model the $L_{s p}-U_{s p}$ transition, which has been described previously $[99,100]$.

In the OBE calculation, the SS state is excited into the final state continuum by coherent two-photon absorption in near resonance with the IP state [Fig. 3(a)]. As the interaction proceeds, the photoexcited bandwidth is wide and encompasses the IP $\leftarrow$ SS transition, but, as the interaction evolves in time, it narrows through destructive interferences [2]; dephasing of the IP-SS coherence populates the IP state, as is observed in the 2PP spectrum in Fig. 2. Thus, the final state signal includes both the coherent two-photon absorption from the SS state as well as photoemission from the IP state. The photon energy is set to separate the spectral features of the $(1+1)$-photon process via the IP state from the coherent two-photon absorption from the SS state; quantum interference caused by the excitation of final states through two differing pathways is negligible [62]. Thus, the calculated 2PP signal predominantly reproduces the rectification of coherence generated by two-photon excitation of the occupied SS state.

In Fig. 3(a) (right), we show constant energy cross sections [interferometric two-pulse correlations (I2PCs)] through the calculated two-pulse interferogram, for the final state energies of the SS and IP states; similar correlation traces oscillating at the driving laser frequency and its second harmonic are observed in the original ITR2PP study of the SS state on $\mathrm{Cu}(111)$ [1], but those measurements do not have the 2D spectroscopic capability. The I2PC signal of the IP state has a slow component due to its incoherent population decay, as is measured in two-color 2PP [86]. The inset in Fig. 3(a) shows an expanded SS component of the ITR-2PP data for time delays that are comparable to or longer than the laser pulse: It is evident that the signal is composed of dominant coherent oscillations at the driving laser frequency $\left(1 \omega_{l}\right)$ and subordinate ones at its second harmonic $\left(2 \omega_{l}\right)[1,48]$. We stress that OBE simulations reproduce the energy- and phase-resolved $\mathrm{mPP}$ measurements of the coherent electron dynamics in 
$\operatorname{Ag}(111)$, as will be evident in experimental results for higher-order processes. We focus on the tilt of interference fringes towards $t=0 \mathrm{fs}$ with an increasing final state energy [inset in Fig. 3(a)], which we show is the prima facie evidence for coherence in a $2 \mathrm{PP}$ process.

To further analyze the calculated ITR-mPP data, we Fourier transform it to generate 2D-FT photoelectron spectra, which correlate the polarization frequencies (energies) that are excited in the sample and appear as coherent oscillations, with final state energies where the $\mathrm{mPP}$ process terminates [Fig. 3(c)]. Local FT features of the $2 \mathrm{D}-\mathrm{FT}$ spectra occur at the $0 \omega_{l}, 1 \omega_{l}$, and $2 \omega_{l}$ energies. The $1 \omega_{l}$ and $2 \omega_{l}$ represent the linear and second-order nonlinear polarization, respectively, of surface electrons of $\mathrm{Ag}(111)$ by the driving field. For a three-level system, $1 \omega_{l}$ and $2 \omega_{l}$ coherences have dominant contributions between the nearest neighboring $\hat{\rho}_{i(i+1)}$ and next-nearest neighboring $\hat{\rho}_{i(i+2)}$ states, respectively [Fig. 1(b)]. The $0 \omega_{l}$, however, represents the envelope function of the interferogram, including the relatively slow incoherent population dynamics of the intermediate state, which is integrated over time in recording the coherent and incoherent contributions to $\mathrm{mPP}$ signal $[48,104,105]$.

Figure 4(a) shows enlarged elements of the 2D-FT photoelectron spectra of the SS state in Fig. 3(c). The polarizations that oscillate at the $1 \omega_{l}$ and $2 \omega_{l}$ frequencies are rectified to produce the final states in the $2 \mathrm{PP}$ process. The polarization ranges cover widths of the driving frequency $\omega_{l}$ and its harmonics. The FT amplitudes of the linear $\left(1 \omega_{l}\right)$ and the second-order nonlinear $\left(2 \omega_{l}\right)$ polarizations tilt up with an increasing final state energy. This tilt is caused by the coherent excitation of a discrete initial state with an ultrafast laser pulse with a broad frequency spectrum, which induces primarily linear polarization oscillations over a broad frequency range (approximately $0.1 \mathrm{eV}$ ) centered on $\omega_{l}$. Likewise, the nonlinear polarizations are also excited and oscillate at $2 \omega_{l}$ and a perturbative series of higher-order harmonics. As sketched in Fig. 4(b), the spectral components of polarizations at $1 \omega_{l}$ or $2 \omega_{l}$ correlate with specific final states, satisfying the energy conservation and energy-time uncertainty. Thus, in a coherent $m$-photon excitation process of a discrete state, slopes of the 2D spectral features are given by $n / m$, where $m$ is the order of photoemission (e.g., $m=2,3,4, \ldots$ ) and $n$ is the order of polarization $\left(n \omega_{l} ; n=1,2, \ldots, n \leq m\right)$ contributing to the signal. For example, for the coherent 2PP process of the SS state of $\operatorname{Ag}(111)$, as described by the three-level system (Figs. 3 and 4), the slopes of the $1 \omega_{l}$ and $2 \omega_{l}$ FT amplitudes are $n / m=1 / 2$ and $2 / 2$, respectively [Fig. 4(a)].

The energy tilting of components of 2D-FT photoelectron spectra is an FT manifestation of the tilting of interference fringes in the time-domain ITR-mPP spectra. Specifically, the tilted interference fringes in the inset in Fig. 3(a) signify that each frequency component of the

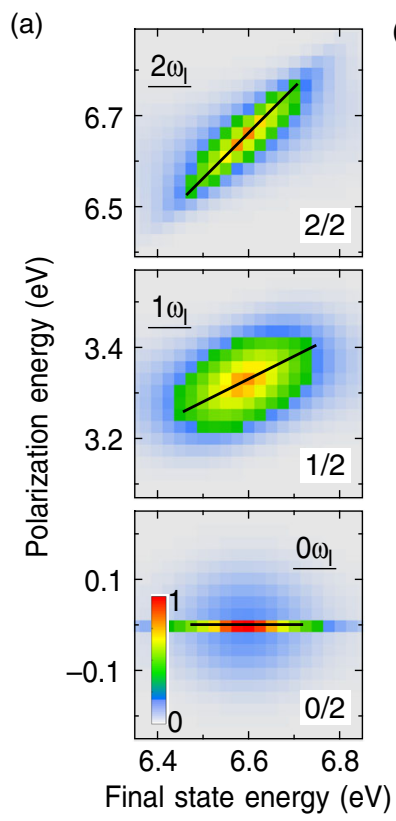

(b)

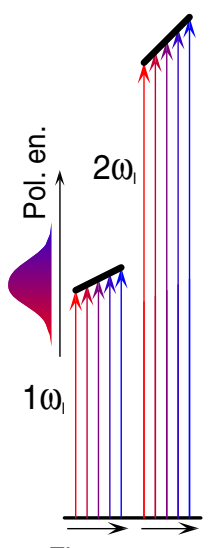

Fin. state en.

FIG. 4. (a) Expanded components of the OBE calculated 2DFT spectra corresponding to the SS state signal from Fig. 3(b). The color table of each polarization order $\left(0 \omega_{l}, 1 \omega_{l}, 2 \omega_{l}\right)$ is scaled separately. The 2D spectra for the zero-order component $\left(0 \omega_{l}\right)$ is horizontal; the $2 \mathrm{D}$ spectra at the driving laser frequency $\left(1 \omega_{l}\right)$ and its second harmonic $\left(2 \omega_{l}\right)$ disperse (tilt) with the final state energy; the slopes for a coherent process are indicated by black lines and quantified by the ratios in the white boxes. (b) Schematic illustration of how the induced polarizations produce the dispersive FT amplitudes in the 2D spectra: Each frequency component within the spectral range of the laser pulse terminates at a specific final state energy; left: linear polarizations $\left(1 \omega_{l}\right)$, right: second-order polarizations $\left(2 \omega_{l}\right)$.

broadband excitation field pulse causes the induced polarization to oscillate at its frequency. Thus, if each frequency component correlates with a specific final state energy, the plot of the final state energy vs the delay time will have tilted interference fringes, as observed; the tilt of the fringes gives insight into optical coherences induced by the laser field in the sample [63]. Similar fringe tilting is also observed in optical spectroscopies that involve interferometric scanning and frequency-resolved optical spectroscopic detection of coherent responses, such as frequency-resolved optical gating or second-harmonic generation [106-108].

In photoemission spectroscopy, however, it is possible that the correlation between the polarization frequency and the final state energy is erased, which happens when the inhomogeneous broadening is large, because populations at different energies oscillating at different frequencies can be excited to the same final state. This case is observed in the ITR-mPP and 2D-FT data from polycrystalline Ta (sample holder; Supplemental Material Figs. S2 and S3 [82]). Likewise, if dephasing is very fast, the polarization frequencies are poorly defined through energy-time 
uncertainty, which broadens the FT spectral components. In such cases, the signal oscillates with the amplitude of the total electric field, rather than its frequency components, because final state energies do not correlate with specific coherences excited in the sample. Consequently, the ITR-mPP fringes do not tilt as is observed, for example, for inhomogeneously broadened polycrystalline samples [54,109].

Next, we extend our discussion to experimentally observed and OBE simulated coherences in the ITRmPP $(m=3,4,5)$ measurements including their ATP components and evaluate them based on the insights from the calculated ITR-2PP data in Fig. 3. By characterizing the interferences in the time and frequency domains, we present a systematic picture of the nonresonant mPP of the SS state as a function of photoemission and polarization order, as well as ATP in the perturbative regime.

\section{B. 3PP and 4PP spectroscopy of $\operatorname{Ag}(111)$}

We measure the 3PP and 4PP spectra of $\mathrm{Ag}(111)$ for photon energies of $\hbar \omega_{l}=1.40$ and $1.60 \mathrm{eV}$ (Fig. 5); in both cases, the SS state is a prominent feature, though it is excited nonresonantly as indicated by the excitation diagram in Fig. 1(a). In this excitation energy range, the dielectric function and the band structure of $\mathrm{Ag}$ show no critical points [110,111]; the excitation energy-dependent electronic responses and differences in the mPP spectra can thus be exclusively attributed to nonresonant photoemission of the order of $m$. Note that, although Ag absorbs light weakly in this spectral region through the Drude absorption, this is a second-order process involving intraband carrier acceleration and scattering to conserve momentum [112], and thus it cannot contribute to the coherent response that we discuss. For $\hbar \omega_{l}=1.60 \mathrm{eV}$ [Fig. 5(a)], photoelectrons are excited from the SS state by a coherent threephoton process. At the final state energy of $\hbar \omega_{l}=1.6 \mathrm{eV}$ above the 3PP spectrum peak of SS, its replica with the same $k_{\|}$dispersion, but with $>100$ times weaker intensity, is also observed. We assign this structure to 4PP, or ATP, of the SS state. In addition, we observe similarly weak 4PP ATP signals from the first IP state and bulk excitation between the $L_{s p}$ and $U_{s p}$. The energies and $k_{\|}$dispersions of the surface states are consistent with the $3 \mathrm{PP}$ and $4 \mathrm{PP}$ processes, as well as with the 2PP data in Fig. 2 and related 2PP literature [39,85,95,100,102].

For $\hbar \omega_{l}=1.40 \mathrm{eV}$ [Fig. 5(b)], four-photon absorption is necessary to observe spectra (4PP) of the SS and IP states, as well as the bulk excitation between the $L_{s p}$ and $U_{s p}$. Again, their replicas are detected at $1.4 \mathrm{eV}$ higher energy, which we also attribute to ATP, i.e., 5PP spectra of the respective states. The $k_{\|}$-resolved ATP data have comparable dispersions as the lower-order processes, and, thus, (a) $\hbar \omega_{1}=1.60 \mathrm{eV}$

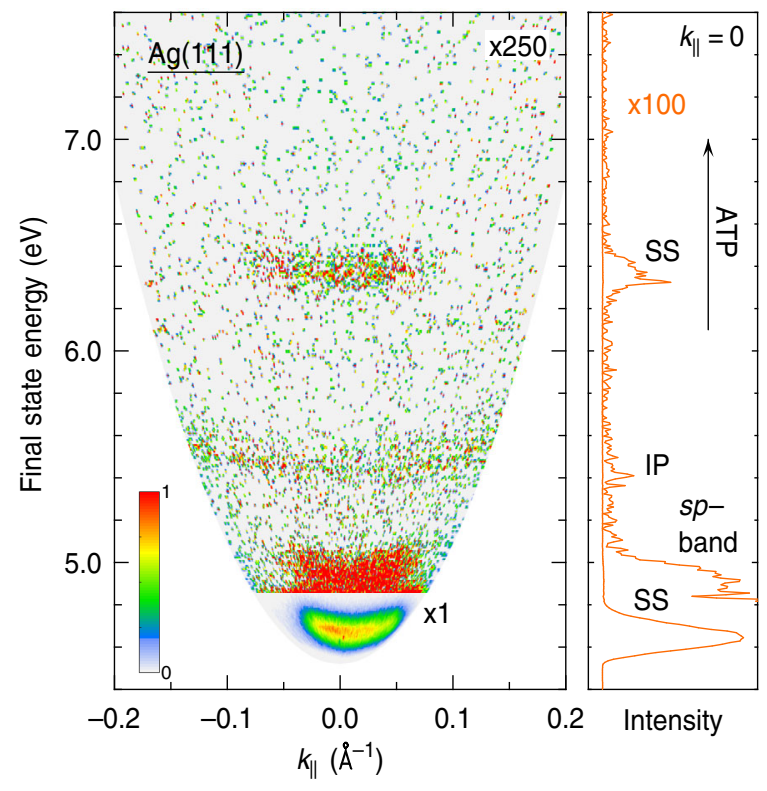

(b) $\hbar \omega_{1}=1.40 \mathrm{eV}$

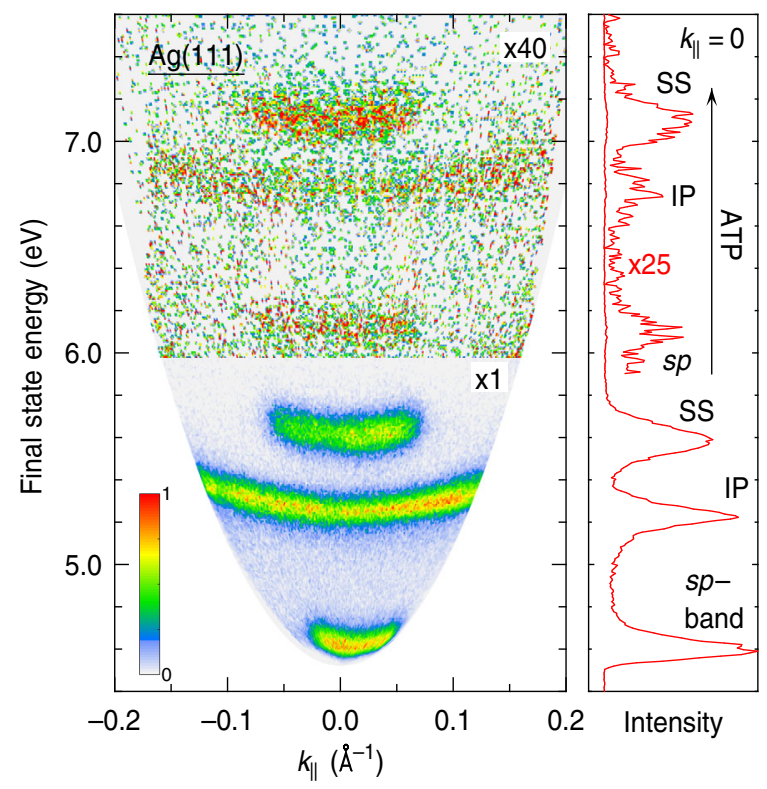

FIG. 5. Energy- and $k_{\|}$-resolved mPP spectra of $\mathrm{Ag}(111)$. Photon energies are chosen such that nonresonant excitation of the SS state is detected (a) by 3PP and (b) by 4PP; photoemission spectral features are labeled in the line profiles taken at $k_{\|}=0$, and excitation diagrams are given in Fig. 1(a). ATP contributions show replicas of states observed in 3PP and 4PP but translated upward by one photon energy. (a) The photoemission spectrum excited with $\hbar \omega_{l}=1.60 \mathrm{eV}$ has a dominant contribution from the SS state excited by 3PP and weaker contributions from the first IP state and the direct $s p$-band transition, which are detected in 4PP. The ATP feature attributed to $m=(3+1)$ excitation is found approximately $1.60 \mathrm{eV}$ above the $3 \mathrm{PP}$ SS state peak. (b) For $\hbar \omega_{l}=1.40 \mathrm{eV}$, the SS state, the first IP state, and the direct $s p$-band transition are detected in 4PP. At higher final state energies, their replicas appear in ATP through $m=(4+1)$ excitation. 
we expect that it involves coherent excitation processes. Significantly, the intensity ratios of the SS and IP states are inverted between the 4PP and 5PP-ATP processes: This inversion implies that in ATP, the highly nonlinear fivephoton absorption process from the SS state is more efficient than the $(3+2)$-photon process via the transiently occupied intermediate IP state.

The nonresonant excitation of $\mathrm{Ag}(111)$ with near-infrared photons is thus an excellent system to study the ultrafast coherent electron dynamics in higher-order mPP and ATP processes; we next present the corresponding ITRmPP data.

\section{Coherent 3PP and 4PP dynamics of the SS state}

The nonresonant excitation of the SS state of $\mathrm{Ag}(111)$ provides a simple system to study high-order, perturbative, coherent electron dynamics systematically for different orders of $m$-photon photoexcitation $(m=3,4)$. In the following, we describe the experimental results for $m=4$ in detail and support our conclusions with results for $m=3$.

In Fig. 6, we show the experimental ITR-4PP data taken with the same experimental conditions as used for the 4PP spectrum in Fig. 5(b) $\left(\hbar \omega_{l}=1.40 \mathrm{eV}, k_{\|}=0\right)$. The I2PC scans at the SS and IP state maxima in Fig. 6 exemplify the coherent dynamics in 4PP; the large peak-to-baseline ratios result from the high nonlinearity of the excitation process. The simultaneous measurement of $\mathrm{mPP}$ over a wide range

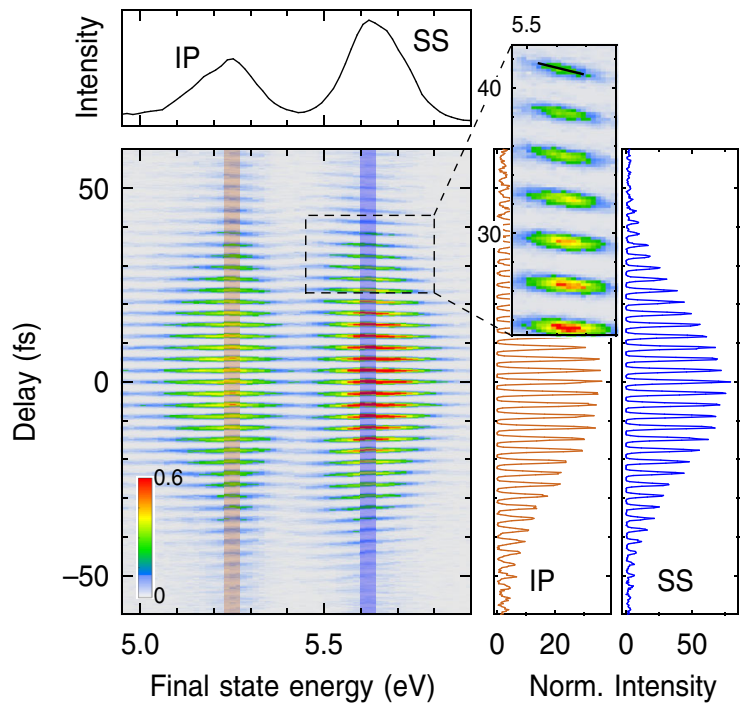

FIG. 6. ITR-4PP experiment on $\mathrm{Ag}(111)$ at a photon energy of $\hbar \omega_{l}=1.40 \mathrm{eV}\left(k_{\|}=0\right)$. Left bottom: Color-coded photoelectron counts as a function of the pump-probe delay and final state energy; top: energy profile taken at $t=0$ fs. Right: I2PC cross sections at the IP and SS final state energies. The high signal-tobaseline ratios are expected for the high-order correlation experiment. Inset: The interference fringes of the SS state tilt towards $t=0 \mathrm{fs}$ with an increasing final state energy; the tilt angle is marked by the black line at approximately $42 \mathrm{fs}$.

of final state energies as a function of the interferometric pump-probe time delay gives further insights into the coherence of the photoemission process: As in the calculated ITR-2PP data in Fig. 3, tilting of the interference fringes towards $t=0 \mathrm{fs}$ with increasing final state energy is observed (inset in Fig. 6). The tilt angles of the interference fringes correlate the induced polarization energy with the detected final state energy.

To analyze the coherent dynamics in more detail, in Fig. 7(a) we plot the SS state components of the 2D-FT photoelectron spectra obtained from the data in Fig. 6. The FT amplitudes for the $0 \omega_{l}-4 \omega_{l}$ components again tilt, respectively, with slopes of $0,1 / 4,2 / 4$, and $3 / 4$ (the $4 \omega_{l}$ signal is too weak to evaluate and probably requires

(a) 4PP Exp.
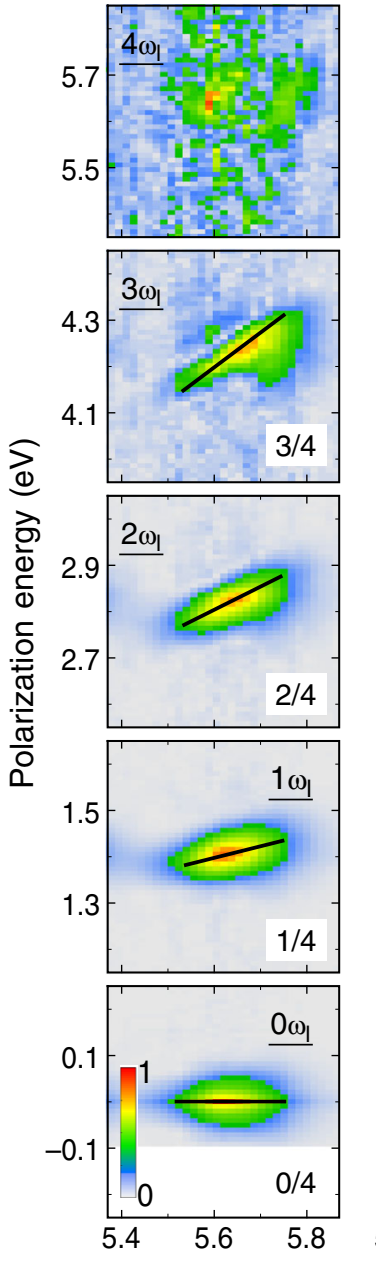

Final state energy $(\mathrm{eV})$

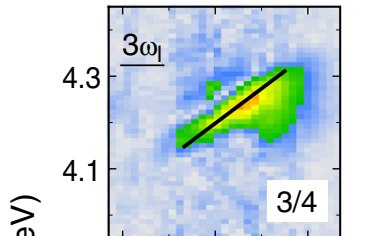

(b) 4PP OBE
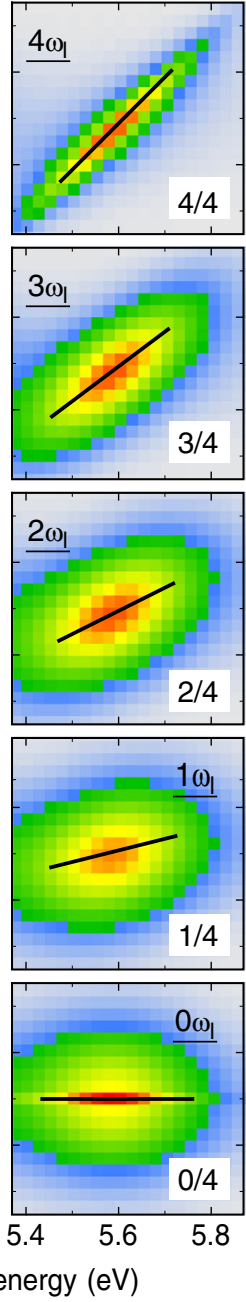

FIG. 7. 2D-FT photoelectron spectral components showing the SS state signal generated by a Fourier analysis (a) of the ITR-4PP experiment in Fig. 6 and (b) a five-level OBE simulation. The color range of each $2 \mathrm{D}$ spectrum is scaled independently. FT amplitudes disperse with final state and polarization energy. The tilt is highlighted by black lines, and their slopes are quantified in the white boxes. 
higher time-resolution). By applying the same arguments as for the 2PP OBE simulation, we explain the slopes with the ratio $n / m$ where now the order of the photoemission process is $m=4$ and of the polarization is an integer multiple of the excitation frequency $(n=0,1,2,3)$. In other words, to achieve 4PP from the SS state, the coherent signal is driven at the harmonics of the driving frequency (numerator $n$ ), and different frequency components of the photoelectron energy correlate with the order of the multiphoton process (denominator $m$ ). To further test this interpretation, we perform an OBE simulation of the 4PP process for a five-level system, which gives comparable 2D-FT spectral components as the experiment [Fig. 7(b)]. The larger widths of the simulated 2D-FT spectra, as compared with the experiment, reflect faster dephasing $1 / T_{2 i j}^{*}$ parameters used in the OBE simulation.

To address the effect of the order $m$ in $\mathrm{mPP}$, we show 2D-FT data for ITR-3PP detection of the SS state in Fig. 8(a) [the energy level diagram is in Fig. 1(a), and

(a) 3PP Exp.

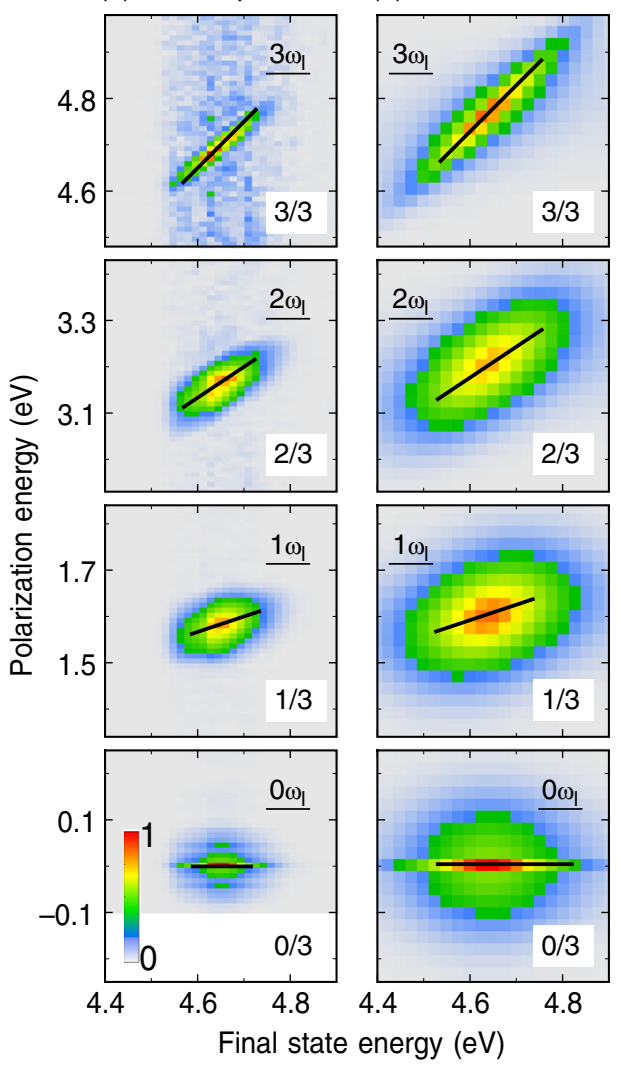

FIG. 8. 2D-FT photoelectron spectral components showing the SS signal generated by a Fourier analysis (a) of the ITR-3PP experiment based on Fig. 5(a) $\left(\hbar \omega_{l}=1.60 \mathrm{eV}, k_{\|}=0\right)$ and (b) a four-level OBE simulation. The color range of each 2D spectrum is scaled independently. FT amplitudes disperse with final state and polarization energy. The tilt is highlighted by black lines, and their slopes are quantified in the white boxes. the 3PP spectrum is in Fig. 5(a) for $\hbar \omega_{l}=1.60 \mathrm{eV}$, $\left.k_{\|}=0\right]$. The ITR-3PP experiments as well as the corresponding four-level OBE simulation [Fig. 8(b)] confirm again the $n / m$ dependence of tilting of the 2D-FT spectra in a nonresonant, coherent process, where now $m=3$ and $n=0,1,2,3$. The 2D-FT photoelectron spectra of the SS state thus systematically depend on the induced polarization signal $(n \leq m)$ and order of the multiphoton excitation process $(m=3,4)$.

\section{Coherent electron dynamics leading to ATP}

The spectra in Fig. 5 show clear contributions of nonresonant photoemission from the SS state following $m$ - and $(m+1)$-photon photoexcitation that produce the $\mathrm{mPP}$ and ATP signals, respectively; the two signal components are recorded simultaneously in an ITR-mPP scan and thus can be compared, even though they differ by a factor of approximately 100 in intensity. Here, we present the first time-resolved measurement of ATP in a solid-state system; the coherent measurement allows us to discuss how it occurs. We first focus on the $(4+1)$ PP ATP process $\left[\hbar \omega_{l}=1.40 \mathrm{eV}\right.$, Figs. 9(a) and 9(b)] and subsequently generalize our observations to the $(3+1)$ PP ATP process $\left[\hbar \omega_{l}=1.60\right.$ eV, Fig. 9(c)]. In Fig. 9(a), we show the ITR$\mathrm{mPP}$ data for the ATP processes from both the first IP and SS states $\left(\hbar \omega_{l}=1.40 \mathrm{eV}, k_{\|}=0\right)$; the I2PC scan at the SS maximum shows a large peak-to-baseline ratio (larger than the IP state) as expected for fifth-order nonlinear processes. Again, we highlight the coherence in the $\mathrm{mPP}$ process of the SS state by showing in the inset in Fig. 9(a) that the interference fringes tilt towards $t=0 \mathrm{fs}$ with an increasing final state energy. The time-domain experiment thus shows that the five-photon excitation of ATP starting from the SS state is a coherent process.

To characterize this coherence, we generate 2D-FT photoelectron spectra of the ATP component [Fig. 9(b)]. FT amplitudes are well structured for $0 \omega_{l}$ (not shown) to $3 \omega_{l}$, but higher-order components have insufficient statistics for analysis. The FT amplitudes show characteristic tilting, like the four-photon excitation in Fig. 7, which in analogy we analyze in Fig. 9(b) with the slope $n / m$, where $m=(4+1)=5$ and $n=1,2$, and 3 for the $1 \omega_{l}, 2 \omega_{l}$, and $3 \omega_{l}$ components.

As a confirmation, we evaluate the 2D-FT data of ATP from the SS state in the $m=(3+1)$ experiment [Fig. 9(c), $\hbar \omega_{l}=1.60 \mathrm{eV}, k_{\|}=0$ ]: FT amplitudes are well resolved for $0 \omega_{l}$ (not shown), $1 \omega_{l}$, and $2 \omega_{l}$, and the slopes are described with $n / m$, where the order of photoemission is $m=(3+1)=4$ and $n=1$ and 2; the FT amplitude of the $3 \omega_{l}$ component is too weak to be evaluated. The analyses of the 2D-FT spectra of ATP in fourth- and fifth-order photoemission are thus consistent and establish the coherent nature of ATP.

The ITR-mPP data show tilted interference fringes and FT amplitudes in the time and frequency domains, where it 
(a) ITR-5PP, ATP

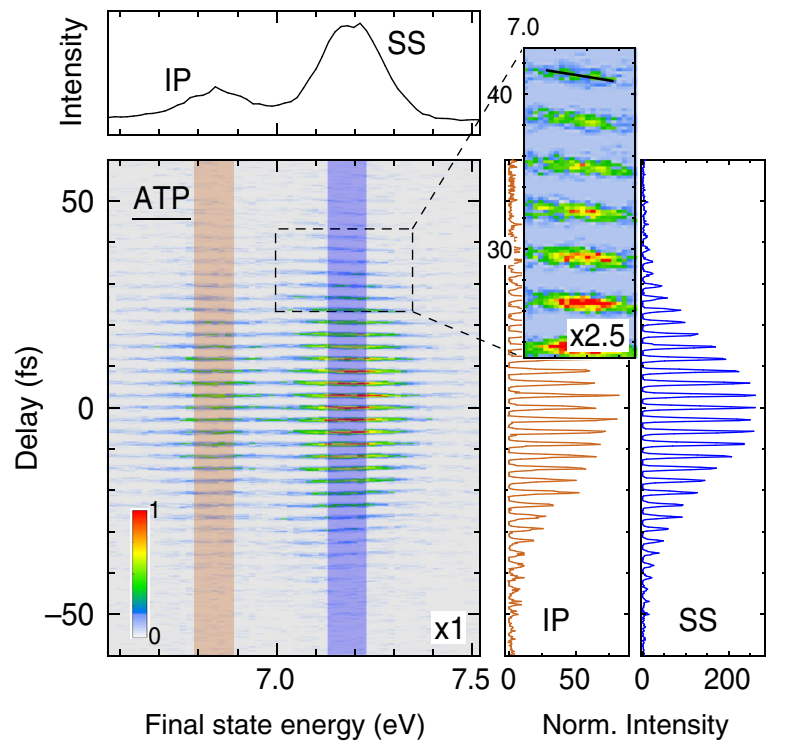

(b) 5PP (ATP), 2D-FT

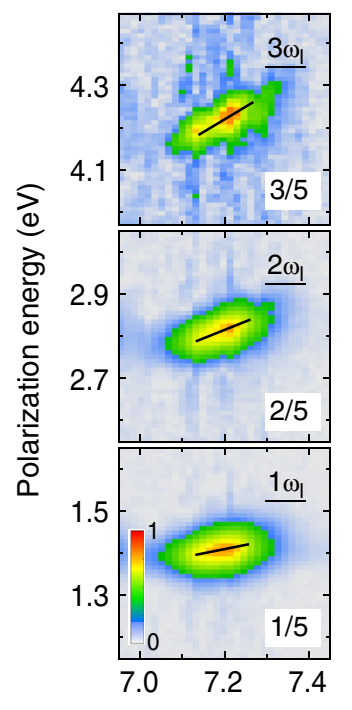

Final state energy (eV) (c) 4PP (ATP), 2D-FT

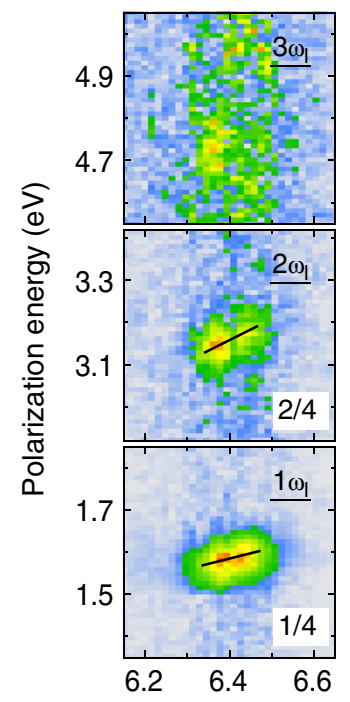

Final state energy $(\mathrm{eV})$

FIG. 9. (a) ITR-mPP experiment for the ATP component of the first IP and SS states that are excited in a $m=(4+1)$-photon process $\left(\hbar \omega_{l}=1.40 \mathrm{eV}, k_{\|}=0\right)$. The data are taken from the same experiment as shown in Fig. 6. Left bottom: Color-coded photoelectron counts as a function of the pump-probe delay and final state energy. Top: Energy profile taken for $t=0$ fs. Right: The cross sections (I2PC scans) of the SS and IP states show a high peak-to-baseline ratio as expected for a fifth-order process. Inset: Tilting of the fringes is highlighted by the black line at $t \approx 41 \mathrm{fs}$. (b) Components of 2D-FT photoelectron spectra of the SS state excited in a $m=(4+1)$ photon ATP process [FT of the data in (a)]. The FT amplitude is observed for $0 \omega_{l}$ (not shown), $1 \omega_{l}, 2 \omega_{l}$, and $3 \omega_{l}$; the slopes are marked with black lines and quantified in the white boxes. (c) Components of 2D-FT photoelectron spectra of the SS state excited in the $m=(3+1)$-photon ATP process $\left(\hbar \omega_{l}=1.60 \mathrm{eV}, k_{\|}=0\right)$; tilted FT amplitude is evident for $0 \omega_{l}$ (not shown), $1 \omega_{l}$, and $2 \omega_{l}$ as marked with black lines and quantified in the white boxes. The color range of each 2D spectra is scaled separately.

is evident that the dominant $\mathrm{mPP}$ and the accompanying $(m+1)$-ATP processes arise from the same physics but in different orders of nonlinearity. Although ATP is usually described as occurring by a sequential process involving first the generation of a photoelectron through mPP, followed by it absorbing one or more photons before emerging from the surface region [6-11], our measurements show that such a process does not dominate. We confirm this conclusion by performing OBE simulations that explicitly distinguish coherent and sequential ATP; the generated 2D photoelectron spectra are shown in Fig. S4, and details on the simulation are given in Supplemental Material [82]. For coherent ATP, in agreement with the results from Figs. 4 and 7-9, tilted FT distribution is detected in the 2D FT spectra. By contrast, our simulations show that, if ATP is a sequential (incoherent) process, a horizontal (flat) FT distribution would result for all orders of polarization, i.e., $0 \omega_{l}, 1 \omega_{l}, 2 \omega_{l}, \ldots$.

In the perturbative regime, the optical field thus drives SS state electrons to oscillate nonlinearly at $m \omega_{l}$, $(m+1) \omega_{l}$, and a perturbative series of higher frequencies. These coherent polarizations (involving the entire electronic system) can produce coherent light emission at the $m$ and $(m+1)$ harmonics of the driving field [70], as has been observed from $\mathrm{Cu}(111)$ for $m=2$ at lower fluences [113]. In addition, the mPP and $(m+1)$ PP ATP signals can be produced by the rectification of components of the induced polarization into photoelectron currents at the $m$ and $(m+1)$ final state energies; our data show this to be a more probable scenario than the $m$-photon final state absorbing an additional photon before departing from the surface. Of course, in a five-photon process, there are many other excitation pathways to reach the final state, but only the rectification of the $(m+1)$ polarization would produce the observed FT amplitude slopes $n / 5$ in Fig. 9(b). That the ATP photoelectron is generated preferentially by the rectification of $(m+1)$ polarization can explain why the intensities of SS and IP states are reversed between the $\mathrm{mPP}$ and ATP orders in Fig. 5(b). Whereas the ATP signal from the SS state results from rectification of the $(m+1)$ polarization, that for the IP state first populates the intermediate state by the rectification of the $3 \omega_{l}$ polarization followed by the rectification of the $2 \omega_{l}$ polarization to generate the ATP signal. Because susceptibilities are time dependent [70], photoemitting an electron of the same order is probably less efficient by a cascaded process of lower-order coherent responses than the direct higher-order process. Although both are driven by an intense electric field, the ATP processes reported here should be contrasted with the nonperturbative regime, where it involves the field-induced recollision of electrons with their atomic cores $[18,114]$. We again emphasize that, under our 
experimental conditions, we observe no spectroscopic evidence of field-induced nonperturbative dynamics.

\section{CONCLUSIONS}

We have elaborated the coherent multiphoton photoemission of the Shockley surface state electrons from Ag (111) surface by means of ITR-mPP experiments and OBE simulations for nonlinear orders of $m=2-5$. The nonresonant energy level climbing of SS electrons in the perturbative regime is dominantly caused by oscillations of the nonlinear polarization at high orders of the driving field, as recorded by ITR-mPP measurements and their Fourier analysis; the coherence can dephase to populate nonresonant intermediate states, such as the IP states, it can be rectified to produce the $\mathrm{mPP}$ signal or, in higher order, its ATP replicas, or it can decay by producing harmonic emission [70]. In time-domain measurements, the coherent electron responses of a metal surface state are evident in the tilting of interference fringes, because the final state energies correlate with the induced polarization frequencies. In the frequency domain, the same physics causes the Fourier amplitudes to tilt such that their slopes are given by the ratio $n / m$, where $n$ is the (non)linear order of the polarization and $m$ is the order of the mPP process. This result stands in contrast with the nonperturbative regime, where the optical field transiently distorts the atomic Coulomb potential and thereby drives electron trajectories through resonances for high harmonic emission.

Our study gives systematic insights into the shapes of 2D photoelectron spectra as obtained by Fourier analysis of ITR-mPP experiments: By addressing the simplest case of the nonresonant excitation of the discrete Shockley surface state of $\operatorname{Ag}(111)$, we establish a benchmark for a deeper understanding of more complex excitation schemes such as in the two-photon IP $\leftarrow$ SS resonant excitation on $\mathrm{Ag}(111)$ [39], the proposed multiexciton generation in organic semiconductors [115], or bulk and surface states in novel materials like topological insulators [116] or As(111) [117]. The coherent excitation of surface states of metals provides a simple electronic structure, which can be easily resolved and selectively excited in an mPP process. With a high-power tunable laser source, the resonant or nonresonant excitations pathways can be selected, excited, and compared. Such coherent excitation of photoemission can be detected in atomic [118], molecular [119], and other solid-state systems where similar experimental procedures and analysis can be applied. Coherent 2D optical spectroscopy has already been applied to excitonic correlations in GaAs [120]. The method reported here provides an alternative approach that probes the induced coherences over a wide energy range with state energy and momentum resolution and that is likely to be equally effective in strongly interacting correlated solid-state materials that may be nonemissive.
We have also reported the first coherent spectroscopic investigation of perturbative ATP processes in solids. Our results show that ATP involving nonresonant excitations can be understood as a consequence of higher nonlinearorder driving of the coherent SS electron oscillations, when compared to the main $\mathrm{mPP}$ signal, rather than the previously envisaged two-step process where a photoelectron is generated and absorbs another photon before emerging from the surface into a vacuum. The energy, $k_{\|}$, and timeresolved ITR-mPP measurements provide insights into coherent optical processes at metal surfaces in the perturbative interaction regime.

\section{ACKNOWLEDGMENTS}

The authors gratefully acknowledge support from DOE-BES Division of Chemical Sciences, Geosciences, and Biosciences Grant No. DE-SC0002313, and M. R. acknowledges support from the Alexander von Humboldt Foundation within the Feodor Lynen Fellowship Program.

[1] S. Ogawa, H. Nagano, H. Petek, and A. P. Heberle, Optical Dephasing in Cu(111) Measured by Interferometric TwoPhoton Time-Resolved Photoemission, Phys. Rev. Lett. 78, 1339 (1997).

[2] H. Petek, A. P. Heberle, W. Nessler, H. Nagano, S. Kubota, S. Matsunami, N. Moriya, and S. Ogawa, Optical Phase Control of Coherent Electron Dynamics in Metals, Phys. Rev. Lett. 79, 4649 (1997).

[3] U. Höfer, I. L. Shumay, C. Reuss, U. Thomann, W. Wallauer, and T. Fauster, Time-Resolved Coherent Photoelectron Spectroscopy of Quantized Electronic States on Metal Surfaces, Science 277, 1480 (1997).

[4] J. Güdde, M. Rohleder, T. Meier, S. W. Koch, and U. Höfer, Time-Resolved Investigation of Coherently Controlled Electric Currents at a Metal Surface, Science 318, 1287 (2007).

[5] C. Attaccalite, M. Grüning, and A. Marini, Real-Time Approach to the Optical Properties of Solids and Nanostructures: Time-Dependent Bethe-Salpeter Equation, Phys. Rev. B 84, 245110 (2011).

[6] W.S. Fann, R. Storz, and J. Bokor, Observation of Above-Threshold Multiphoton Photoelectric Emission from Image-Potential Surface States, Phys. Rev. B 44, 10980 (1991).

[7] F. Banfi, C. Giannetti, G. Ferrini, G. Galimberti, S. Pagliara, D. Fausti, and F. Parmigiani, Experimental Evidence of Above-Threshold Photoemission in Solids, Phys. Rev. Lett. 94, 037601 (2005).

[8] F. Bisio, M. Nývlt, J. Franta, H. Petek, and J. Kirschner, Mechanisms of High-Order Perturbative Photoemission from $\mathrm{Cu}(001)$, Phys. Rev. Lett. 96, 087601 (2006).

[9] F. Bisio, A. Winkelmann, C.-T. Chiang, H. Petek, and J. Kirschner, Band Structure Effects in Above Threshold Photoemission, J. Phys. Condens. Matter 23, 485002 (2011). 
[10] F. Sirotti, N. Beaulieu, A. Bendounan, M. G. Silly, C. Chauvet, G. Malinowski, G. Fratesi, V. Véniard, and G. Onida, Multiphoton k-Resolved Photoemission from Gold Surface States with 800-nm Femtosecond Laser Pulses, Phys. Rev. B 90, 035401 (2014).

[11] M. Aeschlimann, C. A. Schmuttenmaer, H. E. Elsayed-Ali, R. J. D. Miller, J. Cao, Y. Gao, and D. A. Mantell, Observation of Surface Enhanced Multiphoton Photoemission from Metal Surfaces in the Short Pulse Limit, J. Chem. Phys. 102, 8606 (1995).

[12] G. Farkas, C. Tóth, S. D. Moustaizis, N. A. Papadogiannis, and C. Fotakis, Observation of Multiple-Harmonic Radiation Induced from a Gold Surface by Picosecond Neodymium-Doped Yttrium Aluminum Garnet Laser Pulses, Phys. Rev. A 46, R3605 (1992).

[13] D. von der Linde, T. Engers, G. Jenke, P. Agostini, G. Grillon, E. Nibbering, A. Mysyrowicz, and A. Antonetti, Generation of High-Order Harmonics from Solid Surfaces by Intense Femtosecond Laser Pulses, Phys. Rev. A 52, R25 (1995).

[14] F. Krausz and M. Ivanov, Attosecond Physics, Rev. Mod. Phys. 81, 163 (2009).

[15] M. Krüger, M. Schenk, M. Förster, and P. Hommelhoff, Attosecond Physics in Photoemission from a Metal Nanotip, J. Phys. B 45, 074006 (2012).

[16] F. Krausz and M. I. Stockman, Attosecond Metrology: From Electron Capture to Future Signal Processing, Nat. Photonics 8, 205 (2014).

[17] M. F. Ciappina et al., Attosecond Physics at the Nanoscale, Rep. Prog. Phys. 80, 054401 (2017).

[18] M. Krüger, M. Schenk, and P. Hommelhoff, Attosecond Control of Electrons Emitted from a Nanoscale Metal Tip, Nature (London) 475, 78 (2011).

[19] M. Hohenleutner, F. Langer, O. Schubert, M. Knorr, U. Huttner, S. W. Koch, M. Kira, and R. Huber, Real-Time Observation of Interfering Crystal Electrons in HighHarmonic Generation, Nature (London) 523, 572 (2015).

[20] F. Langer et al., Lightwave-Driven Quasiparticle Collisions on a Subcycle Timescale, Nature (London) 533, 225 (2016).

[21] T. L. Cocker, D. Peller, P. Yu, J. Repp, and R. Huber, Tracking the Ultrafast Motion of a Single Molecule by Femtosecond Orbital Imaging, Nature (London) 539, 263 (2016).

[22] P. Hommelhoff and T. Higuchi, Harmonic Radiation from Crystals, Nature (London) 523, 541 (2015).

[23] S. Ghimire, A. D. DiChiara, E. Sistrunk, P. Agostini, L. F. DiMauro, and D. A. Reis, Observation of High-Order Harmonic Generation in a Bulk Crystal, Nat. Phys. 7, 138 (2011).

[24] Y. H. Wang, H. Steinberg, P. Jarillo-Herrero, and N. Gedik, Observation of Floquet-Bloch States on the Surface of a Topological Insulator, Science 342, 453 (2013).

[25] G. Saathoff, L. Miaja-Avila, M. Aeschlimann, M. M. Murnane, and H. C. Kapteyn, Laser-Assisted Photoemission from Surfaces, Phys. Rev. A 77, 022903 (2008).

[26] S. Neppl, R. Ernstorfer, E. M. Bothschafter, A. L. Cavalieri, D. Menzel, J. V. Barth, F. Krausz, R. Kienberger, and P. Feulner, Attosecond Time-Resolved Photoemission from
Core and Valence States of Magnesium, Phys. Rev. Lett. 109, 087401 (2012).

[27] M. Lucchini, L. Castiglioni, L. Kasmi, P. Kliuiev, A. Ludwig, M. Greif, J. Osterwalder, M. Hengsberger, L. Gallmann, and U. Keller, Light-Matter Interaction at Surfaces in the Spatiotemporal Limit of Macroscopic Models, Phys. Rev. Lett. 115, 137401 (2015).

[28] Z. Tao, C. Chen, T. Szilvási, M. Keller, M. Mavrikakis, H. Kapteyn, and M. Murnane, Direct Time-Domain Observation of Attosecond Final-State Lifetimes in Photoemission from Solids, Science 353, 62 (2016).

[29] F. Siek et al., Angular Momentum-Induced Delays in Solid-State Photoemission Enhanced by Intra-Atomic Interactions, Science 357, 1274 (2017).

[30] C. Ropers, D. R. Solli, C. P. Schulz, C. Lienau, and T. Elsaesser, Localized Multiphoton Emission of Femtosecond Electron Pulses from Metal Nanotips, Phys. Rev. Lett. 98, 043907 (2007).

[31] M. Schenk, M. Kruger, and P. Hommelhoff, Strong-Field Above-Threshold Photoemission from Sharp Metal Tips, Phys. Rev. Lett. 105, 257601 (2010).

[32] R. Bormann, M. Gulde, A. Weismann, S. V. Yalunin, and C. Ropers, Tip-Enhanced Strong-Field Photoemission, Phys. Rev. Lett. 105, 147601 (2010).

[33] A. Kubo, K. Onda, H. Petek, Z. Sun, Y. S. Jung, and H. K. Kim, Femtosecond Imaging of Surface Plasmon Dynamics in a Nanostructured Silver Film, Nano Lett. 5, 1123 (2005).

[34] M. Dąbrowski, Y. Dai, and H. Petek, Ultrafast Microscopy: Imaging Light with Photoelectrons on the Nano-Femto Scale, J. Phys. Chem. Lett. 8, 4446 (2017).

[35] M. Aeschlimann, T. Brixner, A. Fischer, C. Kramer, P. Melchior, W. Pfeiffer, C. Schneider, C. Struber, P. Tuchscherer, and D. V. Voronine, Coherent Two-Dimensional Nanoscopy, Science 333, 1723 (2011).

[36] P. Kahl et al., Direct Observation of Surface Plasmon Polariton Propagation and Interference by Time-Resolved Imaging in Normal-Incidence Two Photon Photoemission Microscopy, Plasmonics 13, 239 (2018).

[37] Y. Gong, A. G. Joly, D. Hu, P.Z. El-Khoury, and W. P. Hess, Ultrafast Imaging of Surface Plasmons Propagating on a Gold Surface, Nano Lett. 15, 3472 (2015).

[38] D. Podbiel, P. Kahl, A. Makris, B. Frank, S. Sindermann, T. J. Davis, H. Giessen, M. Horn von Hoegen, and F.-J. Meyer zu Heringdorf, Imaging the Nonlinear Plasmoemission Dynamics of Electrons from Strong Plasmonic Fields, Nano Lett. 17, 6569 (2017).

[39] X. Cui, C. Wang, A. Argondizzo, S. Garrett-Roe, B. Gumhalter, and H. Petek, Transient Excitons at Metal Surfaces, Nat. Phys. 10, 505 (2014).

[40] K. E. Priebe, C. Rathje, S. V. Yalunin, T. Hohage, A. Feist, S. Schäfer, and C. Ropers, Attosecond Electron Pulse Trains and Quantum State Reconstruction in Ultrafast Transmission Electron Microscopy, Nat. Photonics 11, 793 (2017).

[41] Y. Morimoto and P. Baum, Diffraction and Microscopy with Attosecond Electron Pulse Trains, Nat. Phys. 14, 252 (2018). 
[42] H. Petek, H. Nagano, M. J. Weida, and S. Ogawa, Quantum Control of Nuclear Motion at a Metal Surface, J. Phys. Chem. A 104, 10234 (2000).

[43] M. B. Raschke and C. Lienau, Apertureless Near-Field Optical Microscopy: Tip-Sample Coupling in Elastic Light Scattering, Appl. Phys. Lett. 83, 5089 (2003).

[44] P. Hommelhoff, C. Kealhofer, and M. A. Kasevich, Ultrafast Electron Pulses from a Tungsten Tip Triggered by Low-Power Femtosecond Laser Pulses, Phys. Rev. Lett. 97, 247402 (2006).

[45] G. Sciaini and R. J.D. Miller, Femtosecond Electron Diffraction: Heralding the Era of Atomically Resolved Dynamics, Rep. Prog. Phys. 74, 096101 (2011).

[46] A.H. Zewail, Four-Dimensional Electron Microscopy, Science 328, 187 (2010).

[47] A. Feist et al., Ultrafast Transmission Electron Microscopy Using a Laser-Driven Field Emitter: Femtosecond Resolution with a High Coherence Electron Beam, Ultramicroscopy 176, 63 (2017).

[48] H. Petek and S. Ogawa, Femtosecond Time-Resolved TwoPhoton Photoemission Studies of Electron Dynamics in Metals, Prog. Surf. Sci. 56, 239 (1997).

[49] M. Weinelt, Time-Resolved Two-Photon Photoemission from Metal Surfaces, J. Phys. Condens. Matter 14, R1099 (2002).

[50] P. M. Echenique, R. Berndt, E. V. Chulkov, T. Fauster, A. Goldmann, and U. Höfer, Decay of Electronic Excitations at Metal Surfaces, Surf. Sci. Rep. 52, 219 (2004).

[51] X. Y. Zhu, Electronic Structure and Electron Dynamics at Molecule-Metal Interfaces: Implications for MoleculeBased Electronics, Surf. Sci. Rep. 56, 1 (2004).

[52] U. Bovensiepen, H. Petek, and M. Wolf, Fundamentals Dynamics at Solid State Surfaces and Interfaces Vol. 2 (Wiley-VCH Verlag, Weinheim, 2012).

[53] M. Bauer, A. Marienfeld, and M. Aeschlimann, Hot Electron Lifetimes in Metals Probed by Time-Resolved Two-Photon Photoemission, Prog. Surf. Sci. 90, 319 (2015).

[54] S. Tan, A. Argondizzo, C. Wang, X. Cui, and H. Petek, Ultrafast Multiphoton Thermionic Photoemission from Graphite, Phys. Rev. X 7, 011004 (2017).

[55] T. Yamada and T. Munakata, Spectroscopic and Microscopic Investigations of Organic Ultrathin Films: Correlation between Geometrical Structures and Unoccupied Electronic States, Prog. Surf. Sci. 93, 108 (2018).

[56] R. R. Freeman and P. H. Bucksbaum, Investigations of Above-Threshold Ionization Using Subpicosecond Laser Pulses, J. Phys. B 24, 325 (1991).

[57] E. E. B. Campbell, K. Hansen, K. Hoffmann, G. Korn, M. Tchaplyguine, M. Wittmann, and I. V. Hertel, From Above Threshold Ionization to Statistical Electron Emission: The Laser Pulse-Duration Dependence of $\mathrm{C}_{60}$ Photoelectron Spectra, Phys. Rev. Lett. 84, 2128 (2000).

[58] G. Wachter, C. Lemell, J. Burgdörfer, M. Schenk, M. Krüger, and P. Hommelhoff, Electron Rescattering at Metal Nanotips Induced by Ultrashort Laser Pulses, Phys. Rev. B 86, 035402 (2012).

[59] H. Petek, H. Nagano, and S. Ogawa, Hole Decoherence of d Bands in Copper, Phys. Rev. Lett. 83, 832 (1999).
[60] J. Lehmann, M. Merschdorf, W. Pfeiffer, A. Thon, S. Voll, and G. Gerber, Surface Plasmon Dynamics in Silver Nanoparticles Studied by Femtosecond Time-Resolved Photoemission, Phys. Rev. Lett. 85, 2921 (2000).

[61] S. Ogawa, H. Nagano, and H. Petek, Hot-Electron Dynamics at $\mathrm{Cu}(100), \mathrm{Cu}(110)$ and $\mathrm{Cu}(111)$ Surfaces: Comparison of Experiment with Fermi Liquid Theory, Phys. Rev. B 55, 10869 (1997).

[62] S. Ogawa, H. Nagano, and H. Petek, Phase and Energy Relaxation in an Anti-Bonding Surface State: $\mathrm{Cs} / \mathrm{Cu}(111)$, Phys. Rev. Lett. 82, 1931 (1999).

[63] S. Tan, Y. Dai, S. Zhang, L. Liu, J. Zhao, and H. Petek, Coherent Electron Transfer at the Ag/Graphite Heterojunction Interface, Phys. Rev. Lett. 120, 126801 (2018).

[64] B. Gumhalter, Transient Interactions and Coherent Motion of Optically Excited Electron-Hole Pairs in the Image Potential States at Metal Surfaces, Surf. Sci. 518, 81 (2002).

[65] B. Gumhalter, Stages of Hot Electron Dynamics in Multiexcitation Processes at Surfaces: General Properties and Benchmark Examples, Prog. Surf. Sci. 87, 163 (2012).

[66] V. M. Silkin, P. Lazić, N. Došlić, H. Petek, and B. Gumhalter, Ultrafast Electronic Response of Ag(111) and Cu(111) Surfaces: From Early Excitonic Transients to Saturated Image Potential, Phys. Rev. B 92, 155405 (2015).

[67] V. Blanchet, C. Nicole, M.-A. Bouchene, and B. Girard, Temporal Coherent Control in Two-Photon Transitions: From Optical Interferences to Quantum Interferences, Phys. Rev. Lett. 78, 2716 (1997).

[68] W. L. Schaich and N. W. Ashcroft, Theory of Photoemission, Solid State Commun. 8, 1959 (1970).

[69] W. Schattke, Photoemission within and beyond the OneStep Model, Prog. Surf. Sci. 54, 211 (1997).

[70] C. Timm and K. H. Bennemann, Response Theory for Time-Resolved Second-Harmonic Generation and TwoPhoton Photoemission, J. Phys. Condens. Matter 16, 661 (2004).

[71] H. Ueba and B. Gumhalter, Theory of Two-Photon Photoemission Spectroscopy of Surfaces, Prog. Surf. Sci. 82, 193 (2007).

[72] S. T. Cundiff and S. Mukamel, Optical Multidimensional Coherent Spectroscopy, Phys. Today 66, No. 7, 44 (2013).

[73] G. D. Scholes et al., Using Coherence to Enhance Function in Chemical and Biophysical Systems, Nature (London) 543, 647 (2017).

[74] C. M. Li, L. E. Urbach, and H. L. Dai, Second-Harmonic Generation from a Ag(111) Surface at the Interband Transition Region: Role of the Dielectric Function, Phys. Rev. B 49, 2104 (1994).

[75] R. Boyd, Nonlinear Optics (Academic, New York, 2008).

[76] S. Zhang, C. Wang, X. Cui, Y. Wang, A. Argondizzo, J. Zhao, and H. Petek, Time-Resolved Photoemission Study of the Electronic Structure and Dynamics of Chemisorbed Alkali Atoms on Ru(0001), Phys. Rev. B 93, 045401 (2016).

[77] R. G. Brewer and E. L. Hahn, Coherent Two-Photon Processes: Transient and Steady-State Cases, Phys. Rev. A 11, 1641 (1975). 
[78] M. J. Weida, S. Ogawa, H. Nagano, and H. Petek, Ultrafast Interferometric Pump-Probe Correlation Measurements in Systems with Broadened Bands or Continua, J. Opt. Soc. Am. B 17, 1443 (2000).

[79] T. Klamroth, P. Saalfrank, and U. Höfer, Open-System Density-Matrix Approach to Image-Potential Dynamics of Electrons at Cu(100): Energy- and Time-Resolved TwoPhoton Photoemission Spectra, Phys. Rev. B 64, 035420 (2001).

[80] A. Goldmann, V. Dose, and G. Borstel, Empty Electronic States at the (100), (110), and (111) Surfaces of Nickel, Copper, and Silver, Phys. Rev. B 32, 1971 (1985).

[81] C. Homann, C. Schriever, P. Baum, and E. Riedle, Octave Wide Tunable UV-Pumped NOPA: Pulses down to $20 \mathrm{fs}$ at $0.5 \mathrm{MHz}$ Repetition Rate, Opt. Express 16, 5746 (2008).

[82] See Supplemental Material at http://link.aps.org/ supplemental/10.1103/PhysRevX.9.011044 for temperature dependent 2D FT spectra of the SS state component, laser pulse characterization on the polycrystalline Ta sample holder, and additional OBE simulations on the coherent nature of ATP.

[83] L. V. Keldysh, Ionization in the Field of a Strong Electromagnetic Wave, Sov. Phys. JETP 20, 1307 (1965).

[84] T. Hertel, E. Knoesel, M. Wolf, and G. Ertl, Ultrafast Electron Dynamics at Cu(111): Response of an Electron Gas to Optical Excitation, Phys. Rev. Lett. 76, 535 (1996).

[85] S. Ogawa, H. Nagano, and H. Petek, Optical Decoherence and Quantum Beats in $\mathrm{Cs} / \mathrm{Cu}(111)$, Surf. Sci. 427-428, 34 (1999).

[86] M. Marks, C. H. Schwalb, K. Schubert, J. Güdde, and U. Höfer, Quantum-Beat Spectroscopy of Image-Potential Resonances, Phys. Rev. B 84, 245402 (2011).

[87] Y. Wang, E. W. Plummer, and K. Kempa, Foundations of Plasmonics, Adv. Phys. 60, 799 (2011).

[88] V. M. Silkin, A. K. Kazansky, E. V. Chulkov, and P. M. Echenique, Time-Dependent Screening of a Point Charge at a Metal Surface, J. Phys. Condens. Matter 22, 304013 (2010).

[89] G. Raseev, Plasmon Resonances of Ag(001) and Ag(111) Studied by Power Density Absorption and Photoyield, Surf. Sci. 615, 6 (2013).

[90] M. Reutzel, A. Li, B. Gumhalter, and H. Petek, Excitation of Two-Photon Photoemission where Epsilon is near Zero on $A g(111)$, arXiv:1809.02101.

[91] H. Wern, R. Courths, G. Leschik, and S. Hüfner, On the Band Structure of Silver and Platinum from Angle-Resolved Photoelectron Spectroscopy (ARUPS) Measurements, Z. Phys. B 60, 293 (1985).

[92] T. Miller, W. E. McMahon, and T. C. Chiang, Interference between Bulk and Surface Photoemission Transitions in Ag(111), Phys. Rev. Lett. 77, 1167 (1996).

[93] T. Miller and T. C. Chiang, Lineshape Effects in Photoemission from the Valence States of Metals, J. Phys. Condens. Matter 13, 11115 (2001).

[94] F. Roth, T. Arion, H. Kaser, A. Gottwald, and W. Eberhardt, Angle Resolved Photoemission from Ag and Au Single Crystals: Final State Lifetimes in the Attosecond
Range, J. Electron Spectrosc. Relat. Phenom. 224, 84 (2018).

[95] K. Giesen, F. Hage, F. J. Himpsel, H. J. Riess, and W. Steinmann, Two-Photon Photoemission via Image-Potential States, Phys. Rev. Lett. 55, 300 (1985).

[96] R. W. Schoenlein, J. G. Fujimoto, G. L. Eesley, and T. W. Capehart, Femtosecond Relaxation Dynamics of ImagePotential States, Phys. Rev. B 43, 4688 (1991).

[97] R. L. Lingle, Jr., N. H. Ge, R. E. Jordan, J. D. McNeill, and C. B. Harris, Femtosecond Studies of Electron Tunneling at Metal-Dielectric Interfaces, Chem. Phys. 205, 191 (1996).

[98] S. Pawlik, R. Burgermeister, M. Bauer, and M. Aeschlimann, Direct Transition in the System Ag(111) Studied by One- and Two-Photon Photoemission, Surf. Sci. 402-404, 556 (1998).

[99] N. Pontius, V. Sametoglu, and H. Petek, Simulation of Two-Photon Photoemission from the Bulk sp-Bands of Ag (111), Phys. Rev. B 72, 115105 (2005).

[100] A. Winkelmann, V. Sametoglu, J. Zhao, A. Kubo, and H. Petek, Angle-Dependent Study of a Direct Optical Transition in the sp Bands of Ag(111) by One-and Two-Photon Photoemission, Phys. Rev. B 76, 195428 (2007).

[101] S. S. Tsirkin, S. V. Eremeev, E. V. Chulkov, M. Marks, K. Schubert, J. Güdde, and U. Höfer, Temperature Dependence of the Dynamics of the First Image-Potential State on Ag(111), Phys. Rev. B 86, 085424 (2012).

[102] K. Schubert et al., Momentum-Resolved Electron Dynamics of Image-Potential States on Cu and Ag Surfaces, Phys. Rev. B 85, 205431 (2012).

[103] E. Knoesel, A. Hotzel, and M. Wolf, Temperature Dependence of Surface State Lifetimes, Dephasing Rates and Binding Energies on Cu(111) Studied with Time-Resolved Photoemission, J. Electron Spectrosc. Relat. Phenom. 88-91, 577 (1998).

[104] H. Petek, H. Nagano, M. J. Weida, and S. Ogawa, The Role of Auger Decay in Hot Electron Excitation in Copper, Chem. Phys. 251, 71 (2000).

[105] W. Nessler, S. Ogawa, H. Nagano, H. Petek, J. Shimoyama, Y. Nakayama, and K. Kishio, Femtosecond Time-Resolved Study of the Energy and Temperature Dependence of HotElectron Lifetimes in $\mathrm{Bi}_{2} \mathrm{Sr}_{2} \mathrm{CaCu}_{2} \mathrm{O}_{8}^{+}$delta, Phys. Rev. Lett. 81, 4480 (1998).

[106] R. Trebino, Frequency-Resolved Optical Gating: The Measurement of Ultrashort Laser Pulses (Springer, New York, 2000).

[107] A. Anderson, K. S. Deryckx, X. G. Xu, G. Steinmeyer, and M. B. Raschke, Few-Femtosecond Plasmon Dephasing of a Single Metallic Nanostructure from Optical Response Function Reconstruction by Interferometric Frequency Resolved Optical Gating, Nano Lett. 10, 2519 (2010).

[108] M. Mascheck, S. Schmidt, M. Silies, T. Yatsui, K. Kitamura, M. Ohtsu, D. Leipold, E. Runge, and C. Lienau, Observing the Localization of Light in Space and Time by Ultrafast Second-Harmonic Microscopy, Nat. Photonics 6 , 293 (2012).

[109] S. Tan, A. Argondizzo, J. Ren, L. Liu, J. Zhao, and H. Petek, Plasmonic Coupling at a Metal/Semiconductor Interface, Nat. Photonics 11, 806 (2017). 
[110] H. U. Yang, J. D’Archangel, M. L. Sundheimer, E. Tucker, G. D. Boreman, and M. B. Raschke, Optical Dielectric Function of Silver, Phys. Rev. B 91, 235137 (2015).

[111] A. Marini, R. Del Sole, and G. Onida, First-Principles Calculation of the Plasmon Resonance and of the Reflectance Spectrum of Silver in the GW Approximation, Phys. Rev. B 66, 115101 (2002).

[112] N. V. Smith, Classical Generalization of the Drude Formula for the Optical Conductivity, Phys. Rev. B 64, 155106 (2001).

[113] M. J. Weida, S. Ogawa, H. Nagano, and H. Petek, Parallel Excitation Pathways in Ultrafast Interferometric PumpProbe Correlation Measurements of Hot-Electron Lifetimes in Metals, Appl. Phys. A 71, 553 (2000).

[114] T. Higuchi, M. I. Stockman, and P. Hommelhoff, StrongField Perspective on High-Harmonic Radiation from Bulk Solids, Phys. Rev. Lett. 113, 213901 (2014).

[115] W.-L. Chan, M. Ligges, A. Jailaubekov, L. Kaake, L. Miaja-Avila, and X.-Y. Zhu, Observing the Multiexciton
State in Singlet Fission and Ensuing Ultrafast Multielectron Transfer, Science 334, 1541 (2011).

[116] M.Z. Hasan and C. L. Kane, Colloquium: Topological Insulators, Rev. Mod. Phys. 82, 3045 (2010).

[117] P. Zhang et al., Topologically Entangled Rashba-Split Shockley States on the Surface of Grey Arsenic, Phys. Rev. Lett. 118, 046802 (2017).

[118] V. Blanchet, M. Z. Zgierski, T. Seideman, and A. Stolow, Discerning Vibronic Molecular Dynamics Using TimeResolved Photoelectron Spectroscopy, Nature (London) 401, 52 (1999).

[119] C. Z. Bisgaard, O. J. Clarkin, G. Wu, A. M. D. Lee, O. Geßner, C. C. Hayden, and A. Stolow, Time-Resolved Molecular Frame Dynamics of Fixed-in-Space $\mathrm{CS}_{2}$ Molecules, Science 323, 1464 (2009).

[120] K. W. Stone, D. B. Turner, K. Gundogdu, S. T. Cundiff, and K. A. Nelson, Exciton-Exciton Correlations Revealed by Two-Quantum, Two-Dimensional Fourier Transform Optical Spectroscopy, Acc. Chem. Res. 42, 1452 (2009). 